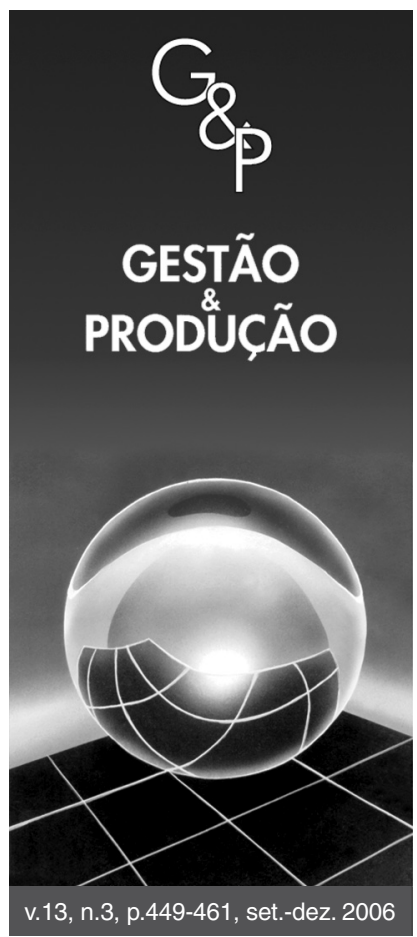

\title{
GESTÃO OPERACIONAL DA COLETA SELETIVA DE RESÍDUOS SÓLIDOS URBANOS - ABORDAGEM UTILIZANDO UM SISTEMA DE APOIO À DECISÃO
}

Eugênio de Oliveira Simonetto,

Centro Federal de Educação Tecnológica de São Vicente do Sul - CEFETSVS, Vinte de Setembro, s/n, CEP 97420-000, São Vicente do Sul, RS, Brasil, e-mail: eosimonetto@cefetsvs.gov.br

Denis Borenstein

Escola de Administração, Universidade Federal do Rio Grande do Sul - UFRGS, Rua Washington Luiz, 855, CEP 90010-460, Porto Alegre, RS, Brasil, e-mail: denisb@ea.ufrgs.br

Recebido em 25/8/2005

Aceito em 26/6/2006

Resumo

O trabalho apresenta a concepção, modelagem e implementação de um sistema de apoio à decisão aplicado ao planejamento operacional da coleta seletiva de resíduos sólidos (SCOLDSS), o qual tem por funcionalidade principal a geração de alternativas ao processo decisório no que se refere à: a) alocação de veículos para a coleta seletiva, bem como determinação do roteiro a ser percorrido; e b) determinação da quantidade diária de resíduos sólidos a ser enviada a cada unidade de triagem, de modo a evitar o desperdício de mão-de-obra e reduzir a quantidade de resíduos enviada aos aterros sanitários. Para seu desenvolvimento, foi utilizada a combinação de técnicas advindas da Pesquisa Operacional, quais sejam: a simulação de eventos discretos e algoritmos/heurísticas para o problema da alocação e roteamento de veículos. Para a validação do SCOLDSS, foram utilizados dados da coleta seletiva de um município do Rio Grande do Sul. É também apresentado um estudo de caso utilizando dados reais da coleta seletiva, no qual são apresentados os resultados gerados pelo sistema, bem como resultados comparados ao sistema atualmente utilizado no processo de coleta seletiva do município.

Palavras-chave: gestão de resíduos sólidos, sistema de apoio à decisão, coleta seletiva.

\section{Introdução}

A reciclagem dos resíduos sólidos é uma alternativa viável para propiciar a preservação de recursos naturais, a economia de energia, a redução de área que demanda o aterro sanitário, a geração de emprego e renda, assim como a conscientização da população para questões ambientais (O’leary et al., 1999). Porém, para um melhor funcionamento, é de vital importância que se implante nas cidades um amplo sistema de coleta seletiva, no qual os recicláveis sejam separados nas residências e coletados pelas municipalidades. Apesar de ser uma excelente alternativa para a redução de resíduos com destino aos aterros, apenas 4,7\% dos resíduos são reutilizados ou reciclados nas cidades gaúchas, segundo a CEMPRE (Organização Não-Governamental Compromisso Empresarial para Reciclagem). Um dos motivos para tal parcela reduzida de reciclagem deve-se ao mau acondicionamento dos resíduos pela população, fato este gerado pela falta de informação acerca da coleta seletiva. Outros fatores que contribuem para o pequeno índice de reciclagem dos resíduos são: a) o alto custo da coleta seletiva para as municipalidades (O'leary et al., 1999; Monteiro et al., 2001); e b) a falta de um sistema corretamente dimensionado em termos da capacidade de armazenamento e processamento de resíduos nas unidades de triagem. Este trabalho tem como principal objetivo apresentar uma ferramenta, bem como resultados gerados por ela, para auxiliar gestores a suplantar estas duas barreiras.

O fluxo dos resíduos provenientes da coleta seletiva constitui-se em um caso particular, pois nas usinas de triagem (ou unidades de triagem) o espaço de armaze- 
namento dos resíduos é reduzido e, dependendo da capacidade de processamento destes, pode-se facilmente ter problemas de alocação de espaço físico. Tal fato é desconsiderado pelos autores da área de logística de resíduos sólidos (Huang et al., 1998; Chang e Wei, 2000; Bhat, 1996; Tung e Pinnoi, 2000), pois quando tratam deste tipo de coleta atribuem capacidade infinita à usina de triagem, como se fosse um aterro sanitário. No caso da distribuição da coleta seletiva de resíduos, não se pode somente buscar a minimização do custo de deslocamentos ou da distância percorrida pelos caminhões, tem-se de levar em consideração a capacidade física e de processamento de resíduos das unidades de triagem de resíduos. É provável que soluções com custo menor de transporte sejam inviáveis, quando se considera a capacidade da unidade de triagem.

Com o desenvolvimento do SCOLDSS, pretende-se auxiliar no planejamento e distribuição da coleta seletiva de resíduos sólidos, buscando, como em outros trabalhos apresentados na literatura (Barlaz et al., 1995; Cunha e Caixeta Filho, 2002; Monteiro et al., 2001; Huang et al., 1998; Chang e Wei, 2000; Bhat, 1996; Tung e Pinnoi, 2000), a redução dos custos de transporte dos resíduos sólidos, mas também objetivando reduzir a quantidade de resíduos recicláveis desperdiçados devido à falta de controle na capacidade de armazenagem e processamento de trabalho nas unidades de triagem deste tipo de resíduo. Desta forma, a principal contribuição desta pesquisa é apresentar uma ferramenta de gestão operacional de resíduos sólidos que leva em consideração a capacidade de processamento das unidades de triagem. Para o desenvolvimento do sistema computacional apresentado no artigo, foram utilizadas técnicas quantitativas oriundas da Pesquisa Operacional, tais como a simulação discreta e algoritmos/heurísticas para a resolução do roteamento de veículos. O uso destas técnicas objetiva agregar qualidade ao processo decisório, pois, muitas vezes, as decisões sobre o planejamento da gestão dos resíduos sólidos são tomadas baseadas somente na experiência dos gestores (Chang e Wei, 2000). Tal fato, segundo estes autores, contribui para o alto custo e o baixo desempenho dos sistemas de coleta de resíduos nas cidades. A utilização de ferramentas de Pesquisa Operacional (PO) na Gestão de Resíduos Sólidos surge como uma alternativa viável para o tratamento da complexidade inerente ao processo de coleta seletiva de resíduos sólidos, pois, por meio do uso destas ferramentas, pode-se representar uma situação do mundo real, estudar seu comportamento (via execução de modelos formais) e tomar decisões com base nas conclusões extraídas. A utilização de técnicas e métodos da PO para o desenvolvimento de estudos na área de coleta de resíduos sólidos já se encontra consolidada (Huang et al., 1998; Chang e
Wei, 2000; Bhat, 1996; Everett e Shahi, 1997; Kulcar, 1996; Tanskanen, 2000; Tung e Pinnoi, 2000; Weintraub et al., 1998).

Portanto, o problema de pesquisa do trabalho desenvolvido consistiu, basicamente, da compreensão do processo de planejamento e execução da coleta seletiva de resíduos sólidos, desde a alocação dos recursos necessários até a distribuição dos resíduos coletados às unidades de triagem, de modo a permitir o desenvolvimento de uma metodologia de apoio à gestão da coleta seletiva (apoiada por ferramenta computacional) que contemplasse os objetivos de: a) minimizar a distância percorrida pelos veículos, bem como o número de veículos alocados; e b) evitar ao máximo o desperdício de resíduos a serem reciclados ou reutilizados, pelo controle da distribuição deles entre as unidades de triagem.

A metodologia de pesquisa adotada para o desenvolvimento do SCOLDSS é a usualmente desenvolvida no âmbito da Pesquisa Operacional (Law e Kelton, 1991), no entanto, com algumas particularidades, por existir na concepção do processo o desenvolvimento de um ambiente computacional para apoio ao processo decisório. $\mathrm{Na}$ etapa de validação do sistema, foi utilizada a técnica de estudo de caso (Yin, 2001).

A metodologia consistiu basicamente das seguintes etapas: 1) estudos exploratórios, em que o problema foi caracterizado e estruturado; 2) desenvolvimento da solução, pela construção de modelos formais capazes de representar o problema; 3) implementação computacional da solução, utilizando-se a tecnologia de sistemas de apoio à decisão (SAD); e 4) validação da solução, por intermédio de testes em laboratório e em campo, para verificar se os resultados obtidos estão de acordo com a realidade observada. A validação foi desenvolvida em um estudo de caso, com a utilização de dados históricos da área de resíduos sólidos recicláveis de Porto Alegre e com a participação de pesquisadores na área. Para o desenvolvimento do SCOLDSS, foi utilizada a arquitetura de sistemas de apoio à decisão proposta por Sprague e Watson (1991), a qual é composta por três subsistemas básicos: banco de dados, modelo decisório e interface, os quais são apresentados na seção 3.

O artigo está organizado da seguinte forma: na seção 2, são apresentados conceitos acerca da reciclagem e da coleta seletiva de resíduos sólidos; na seção 3, é descrito o sistema de apoio à decisão - SCOLDSS -, enfatizando-se sua arquitetura e sua formulação matemática. Algumas questões gerenciais acerca da utilização do sistema de apoio à decisão na gestão da coleta seletiva são discutidas na seção 4. Na seção 5 , são apresentados dados relativos à etapa de validação do sistema; e, por fim, na seção 6, são apresentadas as considerações finais do artigo. 


\section{A reciclagem de resíduos e a coleta seletiva}

Reciclagem, segundo O'Leary et al. (1999), é o processo pelo qual resíduos que são destinados à disposição final são coletados, processados e remanufaturados ou reutilizados. Monteiro et al. (2001) definem reciclagem como sendo a separação de materiais do lixo domiciliar, tais como papéis, plásticos, vidros e outros materiais, com a finalidade de trazê-los de volta à indústria para serem beneficiados. Estes materiais são novamente transformados em produtos comercializáveis.

Segundo Monteiro et al. (2001), a implantação da coleta seletiva é um processo contínuo que é ampliado gradativamente. $\mathrm{O}$ primeiro passo, diz respeito à realização de campanhas informativas de conscientização junto à população, convencendo-a da importância da reciclagem e orientando-a para que separe o lixo em recipientes para cada tipo de material. Posteriormente, deve-se elaborar um plano de coleta, definindo equipamentos, veículos, áreas e a periodicidade de coleta dos resíduos. Finalmente, é necessária a instalação de unidades de triagem para limpeza e separação dos resíduos e acondicionamento para a venda do material a ser reciclado.

Após coletados, os resíduos devem ser transportados para uma unidade de triagem, equipada com lugares para catação, para que seja feita uma separação mais criteriosa dos materiais visando à comercialização deles. É importante que a população seja devidamente orientada para que somente separe como lixo seco os materiais que possam ser comercializados, evitando-se despesas adicionais com o transporte e manuseio de rejeitos, que certamente são produzidos durante o processo de seleção por tipo de material e no enfardamento (Monteiro et al., 2001). Após a implantação da coleta seletiva, o poder público deve manter a população permanentemente mobilizada por meio de campanhas de sensibilização e de educação ambiental (Chang e Wei, 2000). As etapas componentes do processo de coleta seletiva, bem como as decisões inerentes a cada uma destas, podem ser visualizadas na Figura 1.

Etapa

Capacidade de Processamento de Resíduos $\downarrow$

\begin{tabular}{|c|}
\hline Agrupamento Local de Coleta e Unidade de Triagem \\
\hline Alocação de Veículos para a Coleta \\
\hline \\
\hline \\
\hline
\end{tabular}

\section{SCOLDSS - 0 sistema de apoio à decisão proposto}

O sistema de apoio à decisão SCOLDSS foi construído com base nas pesquisas bibliográficas e observações in loco do processo de coleta e distribuição dos resíduos sólidos provenientes da coleta seletiva. Com o desenvolvimento deste sistema, busca-se subsidiar o processo de tomada de decisões operacionais (Goldbarg e Pacca Luna, 2000) dos gestores da área de resíduos sólidos no que se refere à logística dos resíduos sólidos, desde a fase de coleta até a fase de entrega dos resíduos, nas unidades de triagem. Especificamente, o sistema computacional apóia as seguintes tarefas: i) reduzir a quantidade de resíduos sólidos destinada ao aterro sanitário, garantir um percentual de chegada de resíduo em cada unidade de triagem; ii) alocar veículos de coleta; iii) definir o percurso deles; e iv) estimar a capacidade de trabalho (produtividade) das unidades de triagem, em relação à chegada e ao processamento (separação) de resíduos.

Basicamente, o sistema auxiliará a gestão operacional da coleta seletiva pela geração, análise e avaliação de possíveis cenários de operação deste tipo de coleta. Considera-se, neste trabalho, que as etapas para implantação da coleta seletiva (equipamentos, recursos humanos, áreas e periodicidade da coleta seletiva) já estejam previamente definidas.

\subsection{Subsistema banco de dados}

A premissa básica para a construção do subsistema banco de dados do SCOLDSS foi a de selecionar dados de vital importância à geração de informações para os gestores, bem como para alimentar os modelos matemáticos e de simulação existentes no subsistema modelo. Para o desenvolvimento do banco de dados foram utilizados: i) trabalhos realizados anteriormente a este estudo (Huang et al., 1998, Tung e Pinnoi, 2000, Bhat, 1996, Everett e Shahi, 1997; Chang e Wei, 2000); ii) manuais técnicos relativos à área de Gestão de Resíduos Sólidos (O'leary et al., 1999; Monteiro et al., 2001); e iii) entrevistas para levantamento de requisitos junto a especialistas em ges-

\section{Decisão}

Determinar a quantidade de resíduos que cada unidade de triagem pode processar em um dado dia.

Determinar quais locais de coleta enviarão resíduos para unidades de triagens específicas.

Determinar quantos e quais veículos servirão a cada unidade de triagem.

Determinar as rotas de coleta de resíduos, bem como qual veículo deve percorrer cada rota.

Figura 1. Etapas e decisões componentes do modelo decisório. 
tão de resíduos sólidos. O modelo relacional, desenvolvido após a especificação de requisitos do sistema (Booch et al., 2000), do SCOLDSS é apresentado na Tabela 1.

\subsection{Subsistema modelo decisório}

O subsistema modelo decisório do SCOLDSS foi concebido utilizando-se técnicas distintas de modelagem quantitativa: a simulação computacional de eventos discretos e o desenvolvimento de algoritmos/heurísticas para a alocação e roteamento de veículos. A simulação computacional foi utilizada para a determinação das demandas das unidades de triagem, pois elas apresentam um perfil bastante dinâmico de comportamento, basicamente atribuído a sazonalidades e ao perfil de consumo da população. Como o problema da coleta seletiva pode ser modelado como um típico problema de roteamento de veículos com vários depósitos e frota heterogênea, em que os locais de coleta ofertam resíduo sólido a ser demandado por uma unidade de triagem, algoritmos e heurísticas foram desenvolvidos para solucionar este problema.

Primeiro, a determinação da capacidade de processamento de resíduos; segundo, a determinação do escoamento do fluxo de resíduos (veículos e rotas), em conseqüência, do resultado das simulações. Com base na interação da simulação do processamento de resíduos nas unidades de triagem, para a determinação da capacidade de processamento de resíduos em um dia e a execução do problema do roteamento de veículos com múltiplos depósitos e frota heterogênea, são determinados os per- cursos dos veículos de coleta de resíduos, bem como o destino final dos resíduos por eles transportados.

\subsubsection{A integração de técnicas de pesquisa operacional no sistema}

O uso de diferentes técnicas da Pesquisa Operacional é justificado pela natureza distinta dos problemas tratados. A seguir é descrita a estrutura de integração desenvolvida na concepção do sistema de apoio à decisão.

A primeira parte a ser executada durante o processo decisório utilizando-se do sistema é a Simulação de Eventos Discretos no SCOLDSS. O modelo de simulação no SCOLDSS tem como principal finalidade estimar a capacidade de processamento de resíduos sólidos nas unidades de triagem consideradas no processo decisório acerca da coleta seletiva. A determinação da capacidade de processamento é uma particularidade do caso de resíduos potencialmente recicláveis e é originado pelo fluxo de entrada e saída deste tipo de resíduo nas unidades de triagem. Fato este não verificado no caso dos resíduos sólidos que são direcionados ao aterro sanitário, já que não existe a saída dos resíduos sólidos (somente entrada de resíduos) neste tipo de disposição final (Chang e Wei, 2000; Huang et al., 1998; Dmlu, 2004). Para a simulação, utiliza-se o software comercial ARENA (ARENA SOFTWARE, 2003).

O modelo de simulação desenvolvido é composto, basicamente, pelas seguintes variáveis: quantidade de resíduos potencialmente recicláveis a serem coletados;

Tabela 1. Modelo Relacional do SCOLDSS.

\begin{tabular}{|c|c|c|}
\hline Estrutura de Dados & Atributo & Descrição \\
\hline \multirow[t]{4}{*}{ Local de Coleta } & Código do Local & Identificador único do local de coleta \\
\hline & Nome do Local & Descrição do local onde é realizada a coleta \\
\hline & Coordenada X & Representação do ponto $\mathrm{X}$ do local de coleta no mapa \\
\hline & Coordenada Y & Representação do ponto Y do local de coleta no mapa \\
\hline \multirow[t]{4}{*}{ Coleta } & Código do Local & Identificador único do local de coleta \\
\hline & Data/Hora & Data e hora que foi realizada a coleta \\
\hline & Quantidade & Quantidade de resíduos (em kg) coletada no local \\
\hline & Código do Veículo & Identificador único do veículo que realizou a coleta \\
\hline \multirow[t]{5}{*}{ Veículo } & Código do Veículo & Identificador único do veículo de coleta \\
\hline & Placa & Placa do veículo de coleta \\
\hline & Tipo de Veículo & Identificador do tipo de veículo \\
\hline & Código do Depósito & Identificador do depósito, no qual o veículo é alocado \\
\hline & Custo por $\mathrm{Km}$ & Custo por quilômetro rodado do veículo \\
\hline \multirow[t]{3}{*}{ Distância } & Local Origem & Identificador do local de coleta/depósito \\
\hline & Local Destino & Identificador do local de coleta/depósito \\
\hline & Distância & Distância entre os locais em quilômetros \\
\hline \multirow[t]{2}{*}{ Tipo de Veículo } & Tipo de Veículo & Identificador do tipo de veículo \\
\hline & Capacidade & Capacidade de transporte do veículo em quilogramas \\
\hline \multirow[t]{2}{*}{ Depósito (Unidade de triagem) } & Código do Local & Identificador da unidade de triagem \\
\hline & Capacidade Máxima & Capacidade máxima em quilogramas da unidade de triagem \\
\hline
\end{tabular}


quantidade de resíduos armazenados por unidade de triagem; e quantidade de resíduos processados por unidade de triagem. As variáveis aleatórias componentes do modelo de simulação são: taxa média de geração de resíduo por pessoa em área de coleta; taxa média de chegada de resíduos na unidade de triagem; e taxa média de processamento de resíduo em cada unidade.

Para a execução da simulação, a qual determinará a capacidade de processamento de resíduos de cada unidade de triagem, são necessárias as seguintes informações:

- taxa média de geração de resíduos - quantidade média de resíduos gerados pela população. A taxa é expressa em quilogramas por minuto;

- quantidade de resíduos em espera por processamento - quantidade de resíduos aguardando por processamento (triagem) em cada unidade de triagem. Esta informação é expressa em quilogramas; e

- ritmos médios de processamento de resíduos pelos selecionadores - quantidade média de resíduos que cada selecionador é capaz de processar. O ritmo médio dos selecionadores é expresso em quilogramas por minuto.

A identificação das informações anteriormente descritas influenciará na determinação da quantidade total de resíduos processados por dia nas unidades de triagem. A quantidade total de resíduos processados, tanto os resíduos selecionados para retornarem ao mercado, como os resíduos que terão como destino o aterro sanitário, têm de serem levados em consideração pela simulação, pois ambos ocupam espaço físico temporariamente e também consumiram um determinado tempo para serem processados pelos trabalhadores.

Para a distribuição dos resíduos sólidos entre as unidades, durante a simulação, foi utilizado o componente Pickstation do simulador Arena, que seleciona a unidade para enviar a matéria-prima (resíduo) em ordem de precedência, de acordo com o número de recursos (pessoas) utilizado (de modo a evitar ociosidade) e pela quantidade de matéria-prima pós-consumo aguardando para ser processada, pois em cada unidade de triagem é definida uma capacidade máxima de armazenamento de resíduos. Nesta seleção, no modelo de simulação, podem ser consideradas eventuais interrupções no trabalho (almoço, troca de equipe de trabalho) e a variação da produção de um turno para outro.

A informação gerada pela primeira fase do modelo decisório, no caso a simulação do processamento de resíduos nas unidades de triagem, é a demanda de resíduos sólidos (em kg) que cada unidade de triagem é capaz de processar em um determinado dia de trabalho, obtida pela média das $n$ rodadas da simulação.

$\mathrm{Na}$ fase subseqüente da utilização do modelo decisório, têm-se $n$ unidades de triagem (com a demanda já de- finida pela primeira fase do modelo) e m pontos de coleta (a serem percorridos pelos veículos) com oferta de resíduos sólidos recicláveis. Tal descrição denota claramente o problema do roteamento de veículos com vários depósitos (Bodin e Golden, 1981), e, para a resolução, foi utilizada a abordagem proposta por Gillet e Johnson (1974), implementada na forma de heurísticas do tipo "agrupar para depois rotear". Nesta abordagem, primeiramente, devem ser associados pontos de coleta às unidades de triagem específicas, por meio de determinações do tipo "os resíduos do ponto de coleta x são enviados para a unidade de triagem y". Nesta etapa, também é garantido o percentual mínimo de chegada de matéria-prima pósconsumo (em kg) em cada uma das unidades de triagem, de acordo com a capacidade máxima de processamento fornecida pelo simulador.

Quando todos os agrupamentos estiverem formados (locais de coleta associados às unidades de triagem) é aplicada uma heurística para alocação de veículos em tempo de execução do sistema. Como resultado final do processamento desta etapa, tem-se estipulada a quantidade de resíduos que um determinado tipo de veículo $\mathrm{k}$ transportará para uma unidade de triagem i.

A última etapa da execução do sistema consiste da geração das rotas de coleta a serem percorridas, bem como as atribuições de qual veículo deve percorrer cada rota. Após o processamento das rotinas é gerada uma resposta com a seguinte estrutura: para a unidade de triagem $\mathrm{x}, \mathrm{o}$ veículo $\mathrm{n}$ irá percorrer os pontos de coleta $\mathrm{a}, \mathrm{b}$ e c (nesta ordem). Para o desenvolvimento da solução, foi utilizada uma heurística para o problema de roteamento de veículos com frota heterogênea (Renaud e Boctor, 2002), no caso de existência de veículos com capacidades distintas de carregamento. No caso da heurística desenvolvida por Renaud e Boctor (2002), as etapas básicas de processamento são: determinação da ordem de distância em relação à unidade de triagem; geração de rotas, utilizando-se dos diferentes tipos de veículos disponíveis; e seleção da combinação de menor custo dentre as rotas geradas. Em seguida, tenta-se, ainda, melhorar a solução obtida, até se chegar a uma melhor solução, ou permanecer uma já obtida, após $n$ tentativas do procedimento de melhoria. Após o processamento da heurística, os resultados são gerados por meio de mapas com o planejamento diário de coleta.

\subsubsection{Formulação matemática para a aloca- ção e roteamento de veículos}

A formulação matemática do problema de roteamento de veículos não é um problema trivial (Goldbarg e Pacca Luna, 2000). Assim, utilizou-se no desenvolvimento da formulação matemática do modelo decisório, como base inicial, o modelo de Fisher e Jaikumar (1981) para o problema do roteamento de veículos. Posteriormente, 
alterações básicas no modelo original se fizeram necessárias devido às características peculiares ao problema em questão, no caso, a coleta seletiva de resíduos.

Foram identificadas as necessidades de modificações, que, logo após, foram implementadas e agregadas ao modelo básico. As mudanças consistiram, basicamente, no tratamento da existência de mais de uma Unidade de Triagem, ou seja, a inserção de restrições para o tratamento do caso de haver mais de uma unidade de triagem e, a integração de dados originados do modelo de simulação do processamento das unidades de triagem. As restrições para o tratamento do caso da existência de mais de uma Unidade de Triagem e para a integração dos valores da simulação são, respectivamente, as restrições (1) e (6). O modelo e sua descrição são apresentados a seguir no Quadro 1.

\section{Quadro 1. Formulação matemática do modelo decisório} do SCOLDSS.

$$
\begin{aligned}
& \min \sum_{i=1}^{n d} \sum_{j=n d+1}^{n}\left(c_{i j} \sum_{k=1}^{m} x_{i j k}\right) \\
& \text { s.t. } \\
& \sum_{j=n d+1}^{n} l_{i j}-\sum_{j} l_{u j} \leq 0 \\
& i=1, \ldots, n d, u=1, \ldots, n \\
& \sum_{k=1}^{m} y_{i k}=1 \\
& i=n d+1, \ldots, n \\
& \sum_{k=1}^{m} y_{i k}=m \\
& i=1, \ldots, n d \\
& \sum_{k=1}^{m} x_{i j k}=1 \\
& i=1, \ldots, n d, j=1, \ldots, n \\
& \sum_{i=1}^{n d} q_{i} y_{i k} \leq Q_{k} \\
& k=1, \ldots, m \\
& \sum_{i=1}^{n d} q_{i} l_{i j} \leq C_{i} \\
& j=n d+1, \ldots, n \\
& \sum_{i=1}^{n d} q_{j} l_{i j} \geq p C_{i} \\
& j=n d+1, \ldots, n \\
& \sum_{j=n d+1}^{n} x_{i j k}-y_{i k}=0 \\
& \sum_{(i j) \in S} x_{i j k} \leq|S|-1 \\
& i=1, \ldots, n d, k=1, \ldots, m \\
& S \subseteq\{n d+1, \ldots, n\}, k=1, \ldots, m \\
& y_{i k} \in\{0,1\} \\
& i=1, \ldots, n, k=1, \ldots, m \\
& x_{i j k} \in\{0,1\} \\
& i, j=1, \ldots, n, k=1, \ldots, m \\
& l_{i k} \in\{0,1\} \\
& i, j=1, \ldots, n
\end{aligned}
$$

Em que:

$x_{i j k}$ - variável binária que assume o valor 1 quando o veículo k visita o local de coleta j imediatamente após o local de coleta i, 0 em caso contrário;

$l_{i j}$ - variável binária que assume o valor 1 quando um local de coleta $\mathrm{j}$ é associado a uma unidade de triagem $\mathrm{i}$, 0 em caso contrário;

$y_{i k}$ - variável binária que assume o valor 1 se o local de coleta i é visitado pelo veículo k, 0 em caso contrário;

$q_{i}$ - a oferta do local de coleta $\mathrm{i}$;

$Q_{k}$ - a capacidade do veículo k;

$p$ - o percentual mínimo de recebimento de resíduos da demanda máxima da unidade de triagem;

$C_{i}$ - a capacidade de demanda máxima da unidade de triagem i. Este valor depende do resultado da simulação como pode ser visualizado a seguir:

$$
C_{i}= \begin{cases}\mu_{i} t & \text { if } \lambda_{i}>\mu_{i} \\ \lambda_{i} t & \text { otherwise }\end{cases}
$$

$\lambda_{i}$ - o ritmo médio de chegada de resíduo à unidade de triagem;

$\mu_{i}$ - o ritmo médio de processamento de resíduo na unidade de triagem; e

$t$ - o tempo total simulado.

A restrição (1) garante que um local de coleta está associado a somente uma unidade de triagem. A restrição (2) assegura que um determinado veículo passará por cada local de coleta uma única vez. A restrição (3) garante que a unidade de triagem receba uma visita dos veículos associados a ela. Já a restrição (4) dá a garantia de que um determinado veículo pode servir a uma única unidade de triagem; por vezes, a restrição (5) garante que a oferta de um determinado local de coleta não seja maior que a capacidade de carga de um veículo. A restrição (6) assegura que a capacidade de uma unidade de triagem não seja excedida pelas ofertas dos locais de coleta. Nesta restrição ocorre a inserção dos dados provenientes do modelo de simulação do processamento de resíduos. A restrição (7) assegura que uma determinada porcentagem mínima de carga de resíduos irá chegar à unidade de triagem. A restrição (8) garante que os veículos não terão um local de coleta como etapa final das suas rotas, e, sim, uma unidade de triagem (no caso, as distâncias utilizadas correspondem à garagem). Já a restrição (9) tem por finalidade garantir a eliminação de subtours. As restrições (10), (11) e (12) são restrições complementares ao modelo decisório.

No desenvolvimento do SCOLDSS, optou-se pela utilização de abordagens heurísticas, no caso a heurística para roteamento de veículos com cargas heterogêneas proposta por Renaud e Boctor (2002), para a resolução do problema do roteamento de veículos com múltiplos depósitos (Bodin e Golden,1981), ao invés da utilização de programação linear inteira. Tal opção é justificada pela 
grande quantidade de variáveis inteiras componentes do modelo decisório. Um prático exemplo pode ser visualizado em um dia de coleta seletiva, com 30 locais de coleta a serem percorridos, 10 unidades de triagem (atualmente) e 24 veículos (dois turnos por dia) aptos a realizar a coleta. Ou seja, os resíduos coletados em qualquer um destes locais podem ser enviados para qualquer unidade de triagem e podem ser coletados por qualquer um dos veículos disponíveis. Somente neste exemplo, têm-se 14400 variáveis inteiras a serem analisadas pelo sistema, o que, utilizando-se a programação linear inteira, provavelmente, comprometeria seu desempenho computacional. Por tal motivo, utilizou-se a abordagem heurística na implementação do SCOLDSS, de forma a obter um processamento mais rápido do modelo e de evitar possíveis explosões combinatórias no espaço de soluções possíveis.

\subsection{Subsistema Interface (Diálogo)}

Antes da apresentação do processo de desenvolvimento da interface do SCOLDSS, é apresentada uma definição do que se entende por interface, segundo a ênfase do desenvolvimento de um sistema de apoio à decisão. Aprender a usá-los geralmente implica investimento razoável de tempo. Uma boa interface torna a interação com o sistema mais fácil de aprender e usar. Em outras palavras, a interface pode influir, positivamente ou negativamente, na produtividade do usuário, que nem sempre prefere um sistema com mais recursos ou eficiência do ponto de vista computacional.

Para o desenvolvimento do subsistema interface do SCOLDSS, foi levada em consideração a amigabilidade (user-friendly), para faciltar a interação com possíveis usuários finais (gestores da área de resíduos sólidos) que não possuem a obrigação de serem especialistas na área computacional. A interface principal do SCOLDSS é apresentada na Figura 2.

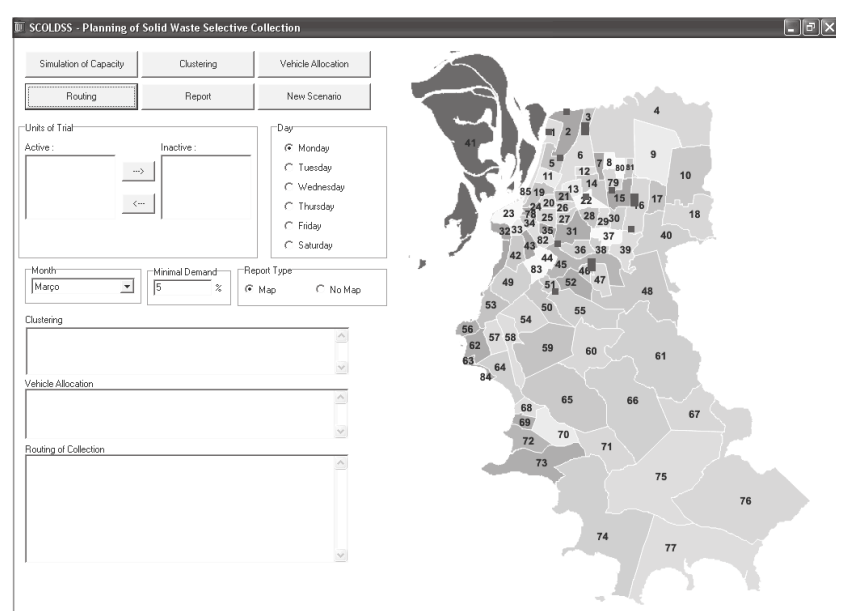

Figura 2. Interface principal do SCOLDSS.hw
Basicamente, o funcionamento da interface, quando da utilização do sistema de apoio à decisão, dá-se da seguinte forma: o usuário informa o dia da semana para o qual é efetuado o planejamento da coleta seletiva, o mês do ano (de forma a considerar as eventuais sazonalidades existentes no processo), as unidades de triagem de resíduos operantes, executando logo a seguir a simulação, para determinar a quantidade de resíduos que cada unidade em operação é capaz de processar diariamente. Posteriormente, o usuário irá executar passo-a-passo as funcionalidades do sistema, quais sejam: a alocação dos veículos; a geração das rotas de coleta; e a geração dos relatórios com os resultados.

\section{O uso do SCOLDSS na gestão da cole- ta seletiva - Um enfoque gerencial}

Esta seção tem por objetivo fundamental apresentar algumas questões gerenciais no processo da coleta seletiva de resíduos sólidos, bem como possíveis cursos de ação, utilizando-se o sistema SCOLDSS para a resolução de tais questões.

\subsection{Etapa 1 - A separação dos resíduos pela população e a coleta pelos veículos}

O sistema de coleta seletiva com separação individualizada dos materiais recicláveis requer considerável espaço para guarda dos contêineres, o que pode inviabilizar sua adoção em apartamentos ou em casas de pequenas dimensões. Nesse modelo, o veículo de coleta pode ter sua carroceria compartimentada de forma a transportar os materiais separadamente. Na maioria das cidades onde existe o sistema, os roteiros de coleta seletiva são realizados semanalmente; caso de Porto Alegre (DMLU, 2004). Já em Seul na Coréia (SEOULIFE, 2003), os resíduos sólidos recicláveis são coletados duas vezes por semana. Para o transporte da coleta, normalmente, utilizam-se caminhões do tipo carroceria aberta ou "gaiola" ou, até mesmo, do tipo "baú" (DMLU, 2004; PREFEITURA SP, 2003).

Nesta primeira parte do processo de coleta, algumas questões gerenciais são de grande importância para o funcionamento e garantia de resultados fidedignos pelo SCOLDSS, a primeira questão refere-se à separação dos resíduos pela população. Basicamente, o perfil de geração de resíduos por cada parte dos habitantes de cada área de coleta é uma informação necessária para o funcionamento do sistema, pois pela média histórica de geração dos resíduos, por local de coleta, é determinada a quantidade de veículos necessária, a ordem em que é efetuada coleta, bem como para qual unidade de triagem deverá ser enviada a quantidade de resíduos coletada. Evidencia-se que, em áreas onde o perfil populacional é de classe mais elevada (classe média e alta), a geração de resíduos reci- 
cláveis é maior, logo assim, pode haver necessidade do sistema alocar dois veículos para efetuar a coleta ou, até mesmo, identificar-se a necessidade de coleta duas vezes por semana. Nas áreas habitadas pelas classes de menor renda, verifica-se que o processo de coleta semanal é satisfatório e que, muitas vezes, em uma única viagem o veículo de coleta é capaz de percorrer até quatro áreas distintas de coleta. Porém, existe um problema maior, de ordem cultural, que diz respeito à não separação dos resíduos recicláveis pela população, para este tipo de problema as campanhas de conscientização de veículos de comunicação e do poder público podem auxiliar no aumento da quantidade de resíduos separados destinados à coleta seletiva.

Uma segunda questão gerencial, inerente à primeira etapa do processo, é a questão referente à falta de veículos de coleta por problemas mecânicos ou pela falta de seus operadores (motoristas e coletores), pois, dependendo da quantidade de veículos ausentes à coleta diária, esta pode ser seriamente comprometida. Quando da alocação de veículos no sistema SCOLDSS, ele identifica em uma base de dados cada um dos veículos disponíveis, o número de viagens que cada veículo pode fazer e, a partir de tais informações, processa a definição das rotas que cada um destes veículos tem de percorrer. No caso da ausência de veículos, o sistema irá efetuar seu processamento e, com muita probabilidade, irá propor alternativa de solução para o processo decisório, desde que se respeite a restrição de capacidade de carga deles. Ou seja, o somatório da quantidade total de resíduos coletados em um dia não poderá exceder o somatório da capacidade de transporte dos veículos coletores.

\subsection{Etapa 2 - Destino final dos resíduos}

Após a coleta, os materiais recicláveis devem ser transportados para uma unidade de triagem, para que seja feita uma separação mais criteriosa dos materiais visando a sua comercialização. As unidades de triagem devem possuir prensas para que os materiais recicláveis, como papéis e plásticos, possam ser enfardados para facilitar a estocagem e o transporte. É importante que a população seja devidamente orientada para que somente sejam separados, como lixo seco, os materiais que possam ser comercializados, evitando-se despesas adicionais com o transporte e manuseio de rejeitos que, certamente, são produzidos durante o processo de seleção por tipo de material e no enfardamento. A distribuição dos resíduos entre as unidades se dá de acordo com a capacidade de recepção de cada unidade, em função de suas dimensões, do número de pessoas envolvidas e do ritmo de trabalho das pessoas envolvidas.

Nesta etapa, uma importante questão gerencial diz respeito à garantia da existência de mão-de-obra nas unidades de triagem para o processamento do material cole- tado. No SCOLDSS, pode-se antecipar, para o processo decisório, quais unidades estão operando e, além disto, se estão operantes, qual sua capacidade de processamento, de acordo com o ritmo de processamento médio de material desenvolvido pelos operadores ativos. Após a informação da capacidade de processamento é que ocorre a simulação da distribuição dos resíduos pelo SCOLDSS.

\section{A validação do SCOLDSS}

O sistema SCOLDSS consiste no desenvolvimento de modelos quantitativos e de simulação para auxílio ao planejamento operacional da coleta seletiva de resíduos sólidos. Como um modelo pode ser definido como uma "representação do mundo real" (Goldbarg e Pacca Luna, 2000), é desejável que o comportamento da representação seja o mesmo (ou mais próximo possível) da realidade em questão, sob determinadas condições especificadas. A este processo denomina-se validação. Na primeira fase de validação (modelo conceitual), foram utilizados dados de artigos científicos e de manuais técnicos referentes à área de gestão integrada de resíduos sólidos, combinados a entrevistas com pesquisadores e gestores da área e, também, observações in loco do processo.

$\mathrm{Na}$ implementação, foi validado cada módulo separadamente e, posteriormente foi desenvolvida a validação integral do SCOLDSS. Dados reais de uma unidade foram utilizados para a validação do módulo de simulação, responsável pela estimativa da capacidade de processamento de resíduos diários das unidades de triagem. Nesta validação, o simulador comportou-se de maneira correta, com um desvio médio de $1,01 \%$ em relação aos dados reais coletados, o que não compromete o desempenho do sistema, tendo em vista que a simulação trata da capacidade máxima de processamento.

A validação de face do SCOLDSS foi desenvolvida com a participação de potenciais usuários do sistema (acadêmicos e profissionais) que, após receberem instruções sobre o funcionamento, o utilizavam com o intuito de verificar a facilidade de uso e a fidedignidade. O principal objetivo da validação de face é alcançar consistência entre as visões do analista do sistema/modelador e a do usuário potencial do modelo de um modo oportuno e efetivo em custo. O SCOLDSS, segundo os usuários, é de fácil usabilidade e apresenta uma significativa contribuição para os gestores da área. Para a execução da validação do SCOLDSS como um todo, foram desenvolvidos estudos de caso com dados reais do processo de coleta seletiva de Porto Alegre RS.

\subsection{Validação do SCOLDSS utilizando da- dos da coleta seletiva de Porto Alegre}

A reciclagem de resíduos no Município de Porto Alegre se dá pela Coleta Seletiva. Tal atividade é de respon- 
sabilidade do Departamento Municipal de Limpeza Urbana e já atinge $100 \%$ da cidade, abrangendo 150 bairros que somados totalizam em torno de sessenta toneladas por dia. $\mathrm{O}$ destino dos materiais colocados à disposição da Coleta Seletiva são as Unidades de Triagem, criadas a partir da necessidade de trabalho de grupos de determinadas áreas carentes da cidade, ex-catadores, papeleiros, populações subempregadas e desempregados, que por meio dessa atividade buscam uma forma de gerar rendimentos, garantindo sua sobrevivência. Todos os rendimentos provenientes da venda dos materiais separados revertem em renda para os recicladores de cada Unidade, que constituem diferentes associações (DMLU, 2004).

Os agentes envolvidos no processo de reciclagem na cidade de Porto Alegre foram identificados por Hiwatashi (1999), são eles: os consumidores, geradores de resíduos; o Departamento Municipal de Limpeza Urbana (DMLU), que realiza a limpeza das ruas e coleta dos resíduos domiciliares; as Unidades de Triagem, associações de trabalhadores, que na verdade, realizam a triagem do material recolhido pelo DMLU; e as empresas de reciclagem, que processam o material pós-consumo nas Unidades de Triagem para poder ser usado como matéria-prima no processo produtivo das empresas de transformação. Os dados da coleta seletiva a respeito de recursos utilizados, percurso médio, capacidade de processamento de resíduos, quantidades coletadas nos locais, distribuição dos resíduos, veículos necessários à validação do SCOLDSS foram fornecidos pela Divisão de Projetos Sociais, Recuperação e Reciclagem (DSR) do DMLU.

Os dados sobre distâncias entre os locais de coletas e as unidades de triagem, assim como as distâncias entre os diferentes locais de coleta foram estimadas utilizando- se o mapa digital oficial de Porto Alegre (PROCEMPA, 2004). Já os dados referentes ao número de associados em cada unidade de triagem foram obtidos em Farah e Barboza (2001).

Para a execução da validação do SCOLDSS, foram selecionadas quinze datas distintas ( 5 em março, 4 em abril e 6 em maio), e foram obtidas significativas melhorias no que se refere às rotas de coleta e ao balanceamento na distribuição dos resíduos coletados. Os dados relativos aos estudos de caso são apresentados nas Tabelas 2 e 3.

$\mathrm{O}$ primeiro aspecto a ser analisado é o que diz respeito ao agrupamento dos locais de coletas feito pelo SCOLDSS. Nos agrupamentos de locais de coleta realizados pelo DMLU, existem casos em que os locais de coleta estão bastante distantes das unidades de triagem, tal fato contribui em muito para o aumento da distância percorrida pelos veículos de coleta. No que se refere ao problema do roteamento de veículos com vários depósitos, foi utilizado o algoritmo proposto por Gillet e Johnson (1974), o qual agrupa os locais de coleta mais próximos às unidades de triagem, desde que estas tenham condições de processar o carregamento.

Outro importante aspecto diz respeito ao uso dos veículos de coleta. Atualmente, o DMLU designa um veículo para cada viagem. Se o local possuir uma taxa elevada de geração de resíduo, enchendo um veículo (acima de 85\% de sua capacidade), tal estratégia é válida. No entanto, não se justifica alocar uma viagem para efetuar a coleta de quatrocentos quilogramas, tendo em vista que a capacidade de transporte dos veículos é de aproximadamente um mil e seiscentos quilogramas. Este fato é bastante comum na atual designação do DMLU. O SCOLDSS busca, se possível, encontrar formas de, em uma única

Tabela 2. Comparação relativa aos percursos entre o sistema atual e o SCOLDSS.

\begin{tabular}{lcccc}
\hline & Percurso Atual & Percurso SCOLDSS & Redução Média de Percurso & Percentual de Redução \\
\hline Segunda-Feira & $546,5 \mathrm{~km}$ & $500,7 \mathrm{~km}$ & $45,8 \mathrm{~km}$ & $8,39 \%$ \\
Terça-Feira & $522,8 \mathrm{~km}$ & $478,4 \mathrm{~km}$ & $44,4 \mathrm{~km}$ & $8,49 \%$ \\
Quarta-Feira & $442,8 \mathrm{~km}$ & $400,4 \mathrm{~km}$ & $42,4 \mathrm{~km}$ & $9,58 \%$ \\
Quinta-Feira & $591,8 \mathrm{~km}$ & $537,2 \mathrm{~km}$ & $54,6 \mathrm{~km}$ & $9,23 \%$ \\
Sexta-Feira & $374,9 \mathrm{~km}$ & $343,1 \mathrm{~km}$ & $31,8 \mathrm{~km}$ & $8,57 \%$ \\
Médias & $495,76 \mathrm{~km}$ & $451,95 \mathrm{~km}$ & $43,8 \mathrm{~km}$ & $8,82 \%$ \\
\hline
\end{tabular}

Tabela 3. Comparação relativa às viagens entre o sistema atual e o SCOLDSS.

\begin{tabular}{lcccc}
\hline & $\begin{array}{c}\text { Número de Viagens } \\
\text { Atual }\end{array}$ & $\begin{array}{c}\text { Número de Viagens } \\
\text { SCOLDSS }\end{array}$ & $\begin{array}{c}\text { Redução Média de } \\
\text { Viagens }\end{array}$ & Percentual de Redução \\
\hline Segunda-Feira & 29 & 23 & 6,0 & $20,71 \%$ \\
Terça-Feira & 27,5 & 24 & 3,5 & $12,7 \%$ \\
Quarta-Feira & 24 & 19 & 5,0 & $20,83 \%$ \\
Quinta-Feira & 33 & 27 & 6,0 & $18,18 \%$ \\
Sexta-Feira & 23 & 19,3 & 3,7 & $17,05 \%$ \\
Médias & 27,36 & 22,53 & 4,83 & $17,89 \%$ \\
\hline
\end{tabular}


viagem, percorrer dois ou três locais de coletas distintos, com o intuito de reduzir o número de viagens e a distância a ser percorrida pelos veículos de coleta. Com a utilização do sistema, pode-se visualizar a redução média de $8,82 \%$ na distância a ser percorrida pelos veículos de coleta e redução de $17,89 \%$ no número de viagens dos veículos por semana.

Considerando-se que a distância média percorrida pelos veículos, atualmente, é de 494,43 km/dia a redução com o uso do SCOLDSS é bastante significativa, já que é estimada em 43,8 km. Por semana, as distâncias percorridas seriam reduzidas, em média, 262,8 km, levando a uma redução anual estimada em $13665 \mathrm{~km}$. Esta redução de quilometragem poderá representar uma economia em torno de US\$10021,88 (cerca de 1\% do orçamento anual do DMLU) por ano, considerando-se custos de operação e manutenção.

No que diz respeito ao número de viagens, a média atual é de 27,3 viagens por dia (163,8 por semana). Com a redução gerada pelo SCOLDSS, este número passaria a ser de 134,9 viagens semanais (redução de 17,89\%), que em um ano resultaria em uma redução de 1502 viagens. Deve-se observar que esta redução é obtida, mesmo considerando-se que o preço médio de uma viagem aumenta de US\$ 6,65 para US\$7,37, utilizando-se os resultados fornecidos pelo SCOLDSS. Este aumento é decorrente do incremento na média de quilometragem percorrida em cada viagem obtida pelo sistema computacional, que, entretanto, aumenta a eficiência global de operação da coleta seletiva. Para tal cálculo, foi considerado somente o consumo de combustível, desconsiderando-se custos de recursos humanos, manutenção de veículos, dentre outros.

Pode-se observar que, nos dias da semana com menos locais de coleta a serem percorridos, são obtidas as taxas mais baixas de melhoria no número de viagens com o uso do SCOLDSS. Tal fato deve-se à redução de soluções no espaço de buscas, ou seja, um número menor de opções para combinações de locais a serem percorridos pelos veículos. Os resultados obtidos evidenciam as melhorias que podem ser realizadas no planejamento operacional do processo de coleta seletiva pela utilização do sistema SCOLDSS, no que se refere à redução da distância percorrida, bem como no total de viagens a serem realizadas para a coleta de resíduos.

Um fator que merece destaque na parte referente à simulação do processamento das unidades de triagem é o que diz respeito à distribuição do material coletado entre as unidades de triagem ativas nos dias de coleta. Constatou-se que, em alguns dias, determinadas unidades de triagem possuíam capacidade de processamento, porém não recebiam nenhuma matéria-prima para ser processada, criando ociosidade no sistema. Ao utilizar-se o percentual de demanda mínima de $6 \%$ na execução das validações, todas as oito unidades de triagem passaram a receber material, porém, sem jamais prejudicar as demais, no que tange às suas capacidades de processamento de resíduos. Desta forma, o aspecto social do programa de coleta seletiva também é beneficiado pelo sistema computacional, garantindo emprego para todos os operários não qualificados das unidades de triagem.

A título de ilustração, é apresentada uma comparação para um cenário específico de operação dentre os diversos realizados. No experimento, referente à Unidade de Triagem 8 (UT8) e 4 viagens de coleta (CT1, CT2, CT3, e CT4), a garagem de onde partem os veículos de coleta é representada por GAR. A estratégia utilizada pela empresa é realizar as viagens de coleta utilizando um veículo para cada local onde é efetuada a coleta seletiva, com as seguintes rotas: GAR- CT1-UT8-GAR, GAR-CT2-UT8GAR, GAR-CT3-UT8-GAR, e GAR-CT4-UT8-GAR. A estratégia gerada pelo SCOLDSS é distinta da utilizada pela empresa, com as seguintas rotas: GAR-CT1-UT8GAR; GAR-CT2-UT8-GAR, e GAR-CT3-CT4-UT8GAR. Na solução atualmente utilizada pelo DMLU, os veículos percorrem, aproximadamente, $78 \mathrm{~km}$, utilizando 4 viagens e, na solução proposta pelo SCOLDSS, o percurso é de aproximadamente $49 \mathrm{~km}$, utilizando 3 . A visualização, utilizando mapas para ilustração dos percursos, é apresentada na Figura 3.

\section{Considerações finais}

O objetivo do trabalho foi apresentar a concepção e o desenvolvimento de um sistema de apoio à decisão para o planejamento operacional da coleta e distribuição dos resíduos sólidos potencialmente recicláveis, levando em consideração as etapas básicas componentes do processo, tais como, a coleta pelos veículos, o destino final após o percurso. A maior contribuição desta pesquisa referese à capacidade do modelo de incorporar o controle da capacidade de armazenamento e de processamento do material nas unidades de triagem deste tipo específico de resíduo. Os resultados possíveis de serem obtidos pelo uso do SCOLDSS são: a) obtenção da redução da distância percorrida pelos veículos de coleta; b) obtenção da redução do número de viagens; e c) obtenção do equilíbrio na distribuição dos resíduos coletados entre as Unidades de Triagem.

Especificamente para o estudo de caso realizado com dados do DMLU/POA, os seguintes resultados foram obtidos:

- Soluções para percursos, em média, 8,82\% melhores que os atualmente obtidos pelo DMLU;

- Redução no número de viagens dos veículos de coleta na ordem de 17,89\%; e 

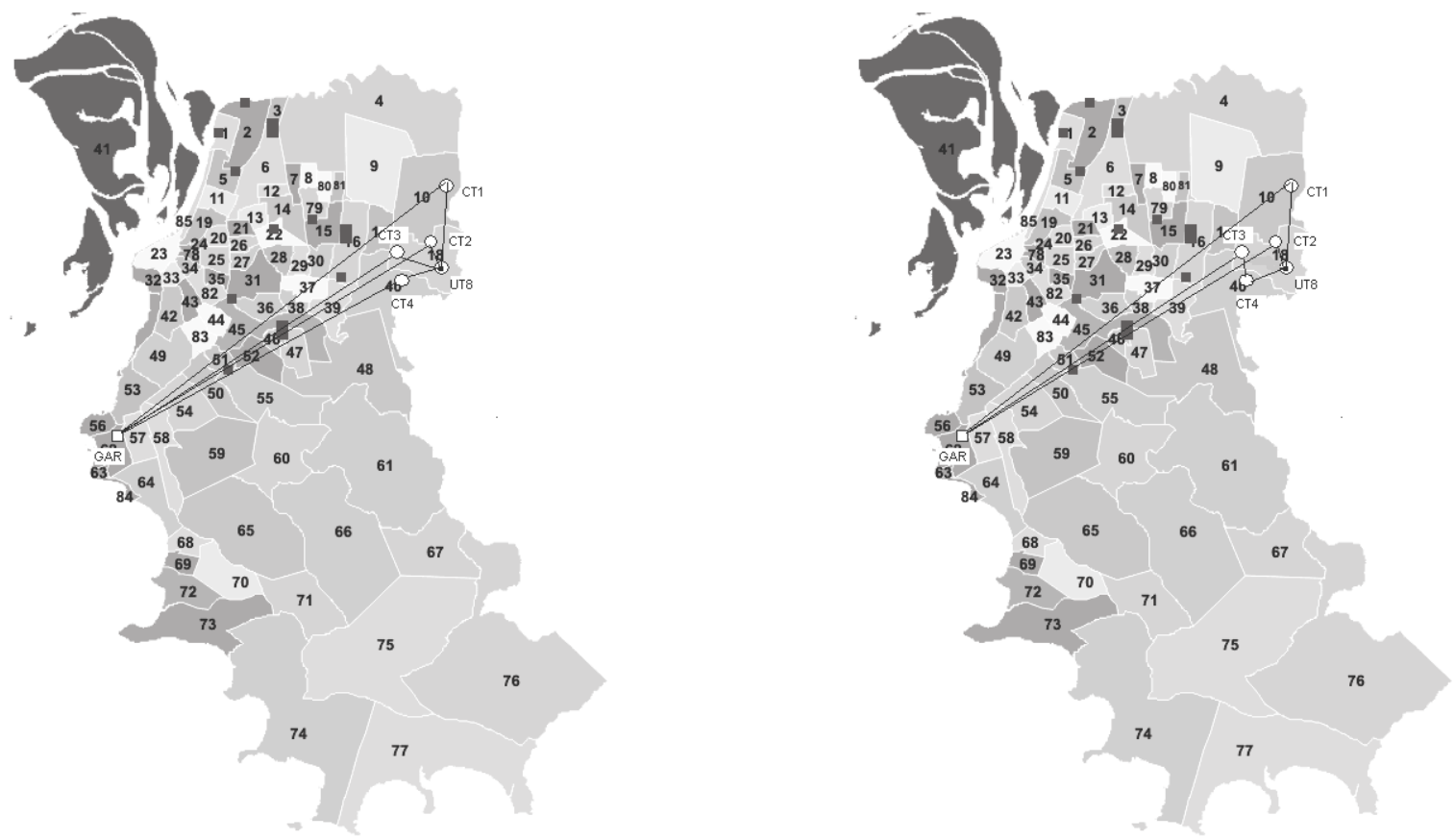

Figura 3. Mapas dos percursos da coleta seletiva utilizando a solução atual e o SCOLDSS.

- Por meio da simulação do processamento de resíduos nas unidades de triagem e da definição do percentual de demanda mínima de resíduos por unidade, pôde-se balancear a distribuição deles entre estas, por dia de coleta. Desta forma, de acordo com a capacidade de processamento individual, é garantido material de trabalho, a cada unidade operante nos dias de coleta seletiva.

Após a análise dos resultados gerados pelo SCOLDSS, uma das recomendações que poderiam ser feitas é a de que, em determinados dias de coleta, seja feito um aumento do número de locais a serem percorridos pelos veículos de coleta. Tal fato verifica-se confrontando o número médio de viagens de coletas proposto pelo SCOLDSS (vinte e duas viagens) com o número de veículos disponíveis para efetuar o serviço (vinte e quatro veículos). Considerando que cada veículo pode efetuar um percurso de coleta pela manhã e outro pela tarde, o total de viagens que podem ser realizadas diariamente é de 48 (quarenta e oito) viagens, ou seja, 26 (vinte e seis) viagens a mais dos que as propostas pelo SCOLDSS. Com o aumento do número de viagens, poder-se-ia ter: a) menor taxa de subutilização dos veículos; b) aumento na quantidade de matéria-prima pós-consumo a ser destinada às unidades de triagem para processamento (tendo em vista que, no atual contexto, estas têm capacidade de processar uma quantidade maior de resíduos); c) aumento na quantidade de resíduos reciclados e reutilizados, gerando maior rentabilidade aos associados; e d) economia de energia e aumento do tempo de vida útil dos aterros sanitários.

Como trabalho futuro, pretende-se desenvolver pesquisas no que se refere ao conhecimento e geração de informações relevantes sobre resíduos sólidos, por meio do desenvolvimento de data warehouses e aplicação de técnicas de data mining em bases de dados sobre resíduos sólidos. O desenvolvimento e aplicação destas técnicas permitirão a identificação de padrões relevantes de comportamento desde a geração dos resíduos até a sua disposição final.

\section{Agradecimentos}

Os autores agradecem à COPESUL e ao CNPq pelo apoio à pesquisa desenvolvida e ao DMLU (DSR) pelo fornecimento dos dados necessários à execução da mesma.

\section{Referências Bibliográficas}

ARENA SOFTWARE. Arena - Overview. Disponível em: $<\mathrm{http} / / / \mathrm{www}$.arenasoftware.com>. Acesso em: $16 \mathrm{de}$ dezembro de 2003.

BARLAZ, M. A. et al. Life-Cycle Study of Municipal Solid Waste Management - System Description. Wa- shington DC-USA: Environmental Protection Agency, 1995.

BHAT, V. N. A model for the optimal allocation of trucks for the solid waste management. Copenhagen-DK. Waste Management \& Research, v. 14, p. 87-96, Jan. 1996. 
BODIN, L. D.; GOLDEN, B. Classification in vehicle routing and scheduling. Networks. USA. v. 11, n. 22, p. 97-108, 1981.

BOOCH, G.; RUMBAUGH, J.; JACOBSON, I. UML Guia do Usuário. Rio de Janeiro: Campus, 2000.

CHANG, N. ; WEI, Y. Siting recycling drop-off in urban area by genetic algorithm-based fuzzy multiobjective nonlinear integer programming modeling. Fuzzy Sets and Systems. Nagoya-JP. v. 114, n. 1, p. 133-149, Aug. 2000.

CUNHA, V. ; CAIXETA FILHO, J. V. Gerenciamento da Coleta de Resíduos Sólidos Urbanos: Estruturação e Aplicação de Modelo Não-Linear de Programação de Metas. Revista Gestão \& Produção, São Carlos-SP, v. 9, n. 2, p. 143-161. Ago.2002.

DMLU-DEPARTAMENTO MUNICIPAL DE LIMPEZA URBANA DE PORTO ALEGRE. Coleta em Porto Alegre. Disponível em: <http://www.portoalegre.rs. gov. $\mathrm{br} / \mathrm{dmlu} /$ coletas.htm $>$. Acesso em: 10 de fevereiro de 2004.

EVERETT, J.W.; SHAHI, S. Vehicle and labor requirements for yard waste collection. Waste Management \& Research. Copenhagen-DK. v. 15, n. 6, p. 627-640, Dec. 1997.

FARAH, M. F.; BARBOZA, H. B (orgs.). 20 Experiências de Gestão Pública e Cidadania. São Paulo:Editora da Fundação Getúlio Vargas, 2001.

FISHER, M.; JAIKUMAR, R. A generalized assignment heuristics for vehicle routing. Networks, v. 11, n. 22, p. 109-124, 1981.

GILLET, B.; JOHNSON, J. Sweep Algorithm for the Multiple Depot Vehicle Dispatch Problem. Proceedings of ORSA/TIMS Meeting, San Juan, Puerto Rico, October 1974.

GILLET, B.; MILLER, L.R. A heuristic algorithm for the vehicle dispatch problem. Operations Research, v. 22, n. 2, p. 340-349, Mar/Apr. 1974.

GOLDBARG, M.C.; PACCA LUNA, H. Otimização combinatória e programação linear. Rio de Janeiro:Campus, 2000.

HIWATASHI, E. O Processo de Reciclagem dos Resíduos Sólidos Inorgânicos Domiciliares em Porto Alegre. 139 f. Dissertação (Mestrado em Administração) - PPGA, Porto Alegre: Universidade Federal do Rio Grande do Sul. 1999.

HUANG, G. H.; BAETZ, B. W.; PATRY, G. G. Trash-Flow Allocation: Planning Under Uncertainty. Interfaces. Hanover-MD-USA. v. 28, n. 6, p. 36-55, Jun. 1998.
KULCAR, T. Optimizing solid waste collection in Brussels. European Journal of Operations Research. BrusselsBE. v. 90, n. 1, p. 71-77, Apr. 1996.

LAW, A. M., KELTON, W. D. Simulation Modeling \& Analysis. $2^{\circ}$ Ed., New York: McGraw-Hill, 1991.

MONTEIRO, J. H. P. et al. Manual de Gerenciamento Integrado de Resíduos Sólidos. Rio de Janeiro: Instituto Brasileiro de Administração Municipal, 2001.

O'LEARY, P. R. et al.. Decision Maker's Guide to Solid Waste Management. v. 2. Washington DC: U.S. Environmental Protection Agency, 1999.

PREFEITURA MUNICIPAL DE SÃO PAULO - SECRETARIA DE SERVIÇOS E OBRAS. Coleta Seletiva. Disponível em: <http://portal.prefeitura.sp.gov.br/secretarias/servicoseobras/projetos/coleta_seletiva/0003>. Acesso em: 10 de fevereiro de 2004.

PROCEMPA - Companhia de Processamento de Dados do Município de Porto Alegre. Mapa Digital Oficial de Porto Alegre. Disponível em: <http://geo,procempa. com.br>. Acesso em: 10 de fevereiro de 2004.

RENAUD, J.; BOCTOR, F. F. A sweep-based algorithm for the fleet size and mix vehicle routing problem. European Journal of Operational Research. Brussels-BE. v. 140, n. 3, p. 618-628, Aug. 2002.

SEOULLIFE. Waste Management - Collection. Disponível em: <http://www.seoulife. com/useful/wast-manage. asp> . Acesso em: 16 de setembro de 2003.

SPRAGUE, R.; WATSON, H. Sistemas de Apoio à Decisão: Colocando a Teoria em Prática. Rio de Janeiro: Campus, 1991.

TANSKANEN, J-H. Strategic planning of municipal solid waste management. Resources, Conservation and Recycling.Utrecht, Netherlands. v. 30, n. 2, p. 111-133, Aug. 2000.

TUNG, D. V. ; PINNOI, A. Vehicle routing-scheduling for waste collection in Hanoi. European Journal of Operational Research. Brussels-BE. v. 125, n. 3, p. 449-468, Sep. 2000.

WEINTRAUB, A.; MARTELL, D.; GUNN, E. Forest Management Challenges for Operational Researchers. European Journal of Operations Research. Brussels-BE. v. 104, n. 1, p. 1-17. Jan,1998.

YIN, R. K. Estudo de Caso, Planejamento e Métodos. Porto Alegre:Bookman, 2001. 


\title{
OPERATIONAL MANAGEMENT OF SOLID WASTE SELECTIVE COLLECTION - AN APPROACH USING DECISION SUPPORT SYSTEM
}

\begin{abstract}
A decision support system (DSS) for modeling and solving the recyclable waste collection operational planning is presented. The computer system has the following objectives: a) to define the vehicles' allocation and routing; $b$ ) to determine the quantity of solid waste to be sent to each waste recyclable trial unit; and c) to generate operational scenarios to be taken into account in the decision process. To accomplish such objectives the DSS employs two well-known operation research techniques, namely simulation and assignment/VRP algorithms. We also present a computational study with real-life data from the solid waste collection in Porto Alegre, Brazil, in which we show that the results provided by the computational system outperforms the operation planning currently adopted.
\end{abstract}

Keywords: solid waste management, decision support system, selective collection. 
